\title{
Deep Learning Image Analysis of Benign Breast Disease to Identify Subsequent Risk of Breast Cancer
}

Adithya D Vellal ${ }^{1}$, Korsuk Sirinukunwattana ${ }^{1,2,3,4}$, Kevin H Kensler ${ }^{5}$, Gabrielle M Baker ${ }^{1}$, Andreea L Stancu ${ }^{1}$, Michael E Pyle ${ }^{1}$, Laura C Collins ${ }^{1}$, Stuart J Schnitt ${ }^{6}$, James L Connolly ${ }^{1}$, Mitko Veta ${ }^{7}$, A Heather Eliassen ${ }^{8,9}$, Rulla M Tamimi ${ }^{8,9,10, *}$ and Yujing J Heng ${ }^{1, \#, *}$

1. Department of Pathology, Harvard Medical School, Beth Israel Deaconess Medical Center, Boston, MA, USA.

2. Institute of Biomedical Engineering (IBME), Department of Engineering Science, Old Road Campus Research Building, University of Oxford, Oxford, UK

3. Big Data Institute, University of Oxford, Li Ka Shing Centre for Health Information and Discovery, Oxford, UK

4. Oxford NIHR Biomedical Research Centre, Oxford University Hospitals Trust, Oxford, UK

5. Division of Population Sciences, Dana Farber Cancer Institute, Boston, MA, USA

6. Dana-Farber/Brigham and Women's Cancer Center, Harvard Medical School, DanaFarber Cancer Institute-Brigham and Women's Hospital, Boston, MA, USA

7. Medical Image Analysis Group, Eindhoven University of Technology, Eindhoven, the Netherlands

8. Channing Division of Network Medicine, Department of Medicine, Harvard Medical School, Brigham and Women's Hospital, Boston, MA, USA

9. Department of Epidemiology, Harvard T.H. Chan School of Public Health, Boston, MA, USA

10. Department of Population Health Sciences, Weill Cornell Medicine, New York, NY, USA

* co-senior authors

\#Corresponding author: Dr. Jan Heng, Department of Pathology, Beth Israel Deaconess Medical Center, Harvard Medical School, 330 Brookline Ave, Dana 517B, Boston, MA 02115, USA; Tel:

+1-617-667-4132; Email: yheng@bidmc.harvard.edu

Running Title: Morphometric analysis in BBD

Conflict of interest statement: The authors declare no conflicts of interest.

Funding: This work was supported by the National Institute of Health/National Cancer Institute R21CA187642 (RMT), R01CA175080 (RMT), R01CA240341 (RMT, YJH), UM1CA186107 (AHE), and U01 CA176726 (AHE), Susan G. Komen for the Cure IIR13264020 (RMT), the Klarman Family Foundation (YJH), BIDMC High School Summer Research Program (ADV), and the Deep Learning 
medRxiv preprint doi: https://doi.org/10.1101/2020.09.02.20186643; this version posted September 3, 2020. The copyright holder for this preprint

(which was not certified by peer review) is the author/funder, who has granted medRxiv a license to display the preprint in perpetuity. It is made available under a CC-BY-NC-ND 4.0 International license.

for Medical Image Analysis research program by Netherlands Organization for Scientific Research and Philips Research P15-26 (MV).

Authors' contributions: Conceived and designed the study: RMT YJH KS. Data analysis: YJH ADV KS MV KHK RMT. Epidemiological data collection: RMT KHK. Breast pathology expertise: GMB LCC SJS JLC. Computational method and data acquisition: ADV KS ALS MEP YJH. All authors contributed to the writing and reviewing of the manuscript. 


\begin{abstract}
Background

New biomarkers of risk may improve breast cancer risk prediction. We developed a computational pathology method to segment benign breast disease (BBD) whole slide images (WSIs) into epithelium, fibrous stroma, and fat. We applied our method to the BBD breast cancer nested case-control study within the Nurses' Health Studies to assess whether computer-derived tissue composition or a morphometric signature was associated with subsequent risk of breast cancer.
\end{abstract}

\title{
Methods
}

Tissue segmentation and nuclei detection deep-learning networks were established and applied to 3795 WSIs from 293 cases who developed breast cancer and 1132 controls who did not. Percentages of each tissue region were calculated and 615 morphometric features were extracted. Elastic net regression was used to create a breast cancer morphometric signature. Associations between breast cancer risk factors and age-adjusted tissue composition among controls were assessed using analysis of covariance. Unconditional logistic regression, adjusting for the matching factors, BBD histological subtypes, parity, menopausal status, and BMI evaluated the relationship between tissue composition and breast cancer risk.

\section{Results}

Among controls, BBD subtypes, parity, and number of births were differentially associated with all three tissue regions $(p<0.05)$; select regions were associated with childhood body size, BMI, age of menarche, and menopausal status $(p<0.05)$. Higher proportion of epithelial tissue was associated with increased breast cancer risk (OR=1.39, 95\% $\mathrm{Cl}$ 0.91-2.14 comparing highest and lowest quartiles; $p$-trend<0.05). No morphometric signature was associated with breast cancer.

\section{Conclusion}

The amount of epithelial tissue may be incorporated into risk assessment models to improve breast cancer risk prediction. 


\section{Introduction}

Breast cancer is the most common cancer in women. One in eight women in the United States will develop breast cancer in her lifetime ${ }^{1}$. Whilst early detection is imperative, identifying and lowering breast cancer risk may help reduce breast cancer morbidity and mortality. Breast cancer risk factors may be non-modifiable (e.g., genetics, dense breast tissue, and benign breast disease (BBD)) or modifiable (e.g., adiposity and alcohol consumption). Researchers continue to identify new biomarkers of risk ${ }^{2-7}$ as well as update risk assessment models ${ }^{8-12}$ to improve breast cancer risk prediction.

Technological advances have enabled the engineering of deep-learning algorithms to analyze whole slide images (WSIs) for disease detection and diagnosis ${ }^{13-18}$, including discriminating between breast cancer and benign breast tissue ${ }^{19-21}$. Our group recently developed a novel deep-learning based computational pathology method to capture quantitative measures of breast terminal duct lobular unit (TDLU) involution ${ }^{22,23}$, a purported biomarker of breast cancer. We reported that neither quantitative measures of TDLU involution derived by our technology nor traditional manual qualitative assessment by pathologists was associated with breast cancer among women diagnosed with $\mathrm{BBD}^{24}$.

In this manuscript, we engineered another deep-learning based computational pathology method to 1) segment BBD histopathological images into epithelial, fibrous stroma, and fat regions; 2) calculate the amount of each tissue region expressed as a percentage of 
medRxiv preprint doi: https://doi.org/10.1101/2020.09.02.20186643; this version posted September 3, 2020. The copyright holder for this preprint

(which was not certified by peer review) is the author/funder, who has granted medRxiv a license to display the preprint in perpetuity. It is made available under a CC-BY-NC-ND 4.0 International license.

total tissue; and 3) extract morphometric features from each tissue region. We applied our method to the BBD breast cancer nested case-control study within the Nurses' Health Study (NHS) and NHSII to evaluate whether computer-derived tissue composition or a morphometric signature in women diagnosed with BBD was associated with subsequent risk of breast cancer. 


\section{Materials and Methods}

\section{Study population}

The Nurses' Health Study (NHS) and NHSII were established in 1976 and 1989, with 121,700 US female registered nurses aged 30-55 years and 116,429 nurses aged 25-42 years, respectively. NHS/NHSII participants first completed baseline questionnaires that provided a medical history as well as extensive information about demographic, lifestyle, reproductive, and dietary risk factors for breast cancer ${ }^{25}$. In biennial follow-up questionnaires, participants provide updated information and report new diagnoses of BBD or breast cancer. Participants who reported a diagnosis of BBD were contacted for consent to retrieve pathology records and BBD lesion specimens for centralized pathology review ${ }^{4,26,27}$. Participants who reported breast cancer were confirmed verbally by the participant, via medical record review, or via the cancer registry. Eligible women with biopsy-confirmed BBD were placed into two sub-studies within the NHS/NHSII-the BBD Incidence study ${ }^{28-31}$ and/or the BBD breast cancer nested casecontrol (NCC) study ${ }^{2-4,24,26,27,32-35}$. Whole slide images (WSIs) from women in the BBD Incidence study were used in the development phase to engineer the deep-learning networks. The BBD NCC study was used in the application phase to determine if computer-derived tissue composition or morphometric features were associated with subsequent breast cancer.

Development Phase: Engineering the tissue segmentation network

Forty-eight hematoxylin and eosin (H\&E) histopathological slides from the BBD Incidence study were selected and digitized at 40x magnification (Panoramic SCAN 150, 
3DHISTECH Ltd, Budapest, Hungary). These 48 WSIs encompassed a wide variety of epithelial morphology from normal epithelium (i.e., TDLUs; $n=6$ )) to non-malignant BBD lesions classified into three histopathological subtypes. The non-proliferative subtype was represented by cysts $(n=5)$ and fibroadenoma $(n=5)$. Proliferative without atypia subtype included intraductal papilloma $(n=5)$, radial scars $(n=5)$, adenosis $(n=5)$, columnar cell change $(n=4)$, and usual ductal hyperplasia $(n=5)$. Proliferative with atypia lesions consisted of atypical ductal hyperplasia $(n=2)$, atypical lobular hyperplasia $(n=4)$, and flat epithelial atypia $(n=2)$. For each WSI, four patches of $2048 \times 2048$ pixels were selected with at least one patch containing the lesion. Each patch was first manually annotated by a researcher for epithelium, fibrous stroma, fat, and background, and later manually verified by a board-certified pathologist (Supplementary Figure 1). The 48 WSIs were split into training and testing sets in a 3:1 ratio. Supplementary Figure 2A displays examples of patches used to train the network.

To segment BBD images into background, epithelial (normal TDLUs, TDLUs exhibiting proliferative or metaplastic changes, and various BBD lesions), fibrous stroma (inter- and intralobular), and fat regions, we engineered a custom 21-layer fully convolutional network inspired by VGG-Net ${ }^{36}$ and U-Net ${ }^{37}$ (Supplementary Table 1 ). This network operated at a magnification level of $2.5 \times$, using the sliding window approach ${ }^{38}$ with an input size of $128 \times 128$ pixels to perform segmentation. To ensure that the network focused on differentiating tissue types by texture and was indifferent to variations in staining, data augmentation in the form of color normalization, random color and intensity shifts and geometrical image transformations were 
performed during training. Patches were flipped vertically and horizontally, transposed, and altered in hue, brightness, contrast, and saturation prior to every iteration.

Training was completed in two steps, using an active learning process ${ }^{39}$. The network was first trained using a complete training set consisting of over 35,000 patches. Uncertainty measure was computed for each training patch through pixel-wise margin sampling ${ }^{40}$. The 7,500 patches with the highest certainty and the 7,500 patches with the lowest certainty were then used to construct a smaller training set. This new 15,000 patch training set was used to retrain the network. This training methodology was utilized in order to prevent the inclusion of ambiguously annotated patches in the training set. The log-likelihood function was used as a loss measure. In order to mitigate class imbalance, each term of the loss function was normalized based on the distribution of the corresponding tissue type at a patch-by-patch level. Additionally, each class was weighted with empirically determined coefficients to achieve the best overall model performance based on the test set. The background weight was 0.7 , epithelium weight was 0.9 , fibrous stroma weight was 1.4 , and fat weight was 1.1 . Adaptive moment estimation optimization ${ }^{41}$ with an initial learning rate of $10^{-4}$ and batch size of eight was used throughout training.

Development Phase: Engineering the cell nuclei detection network

One of the goals of this computational method was to extract morphometric features from tissue regions. Some of these morphometric features require additional information from cell nuclei. Thus, a nuclei detection network was also created using a set of $30 \mathrm{H} \& \mathrm{E}$ breast 
cancer WSIs from The Cancer Genome Atlas where cell nuclei had been previously annotated ${ }^{42}$. Nuclei detection was performed using a fully convolutional U-Net architecture ${ }^{37}$. Images were segmented into nucleus, nuclei membrane, and background, with the nuclei membrane label being established to ensure proper separation of overlapping nuclei. Any pixel labeled as nuclei membrane by the network was re-classified as part of the background before generating the final binary nuclei mask. This network segmented nuclei at 10x magnification and used the sliding window approach ${ }^{38}$, with an input size of $144 \times 144$ pixels for segmentation. While no two-stage training scheme was employed with this model, the details of the color normalization, data augmentation, training, and loss function steps were similar to the tissue segmentation network. The two notable differences were that the log-likelihood loss function was not additionally weighted by class, and each training batch consisted of 32 patches rather than eight. See Supplementary Figure 2B for example patches used for training this network. All networks were developed using the Tensorflow 0.12.0 API for Python 2.7. The source code for our networks is available at https://github.com/avellal14/BBD_Pipeline.

\section{Development Phase: Statistical Analysis}

To evaluate the tissue segmentation network, precision, recall, and Dice similarity coefficient were calculated using the held-out test set $(n=48)$. Dice similarity coefficient is the harmonic mean of precision (i.e., sensitivity) and recall (i.e., positive predictive value) and assesses how accurate the automated segmentation compares with ground truth on a pixelwise basis. The range for Dice similarity coefficient is from 0 to 1 , with 1 indicating perfect 
overlap. For nuclei detection, three-fold cross validation was used to obtain the precision, recall, and Dice similarity coefficient of the network.

Application Phase: BBD NCC study participants

The study protocol was approved by the institutional review boards of the Brigham and Women's Hospital and Harvard T.H. Chan School of Public Health, and those of participating registries as required. The BBD NCC study consisted of 293 cases and 1132 controls (total $n=1425$ ). Cases reported a diagnosis of invasive breast cancer after the cohort baseline (through 1998 for NHS, through 1999 for NHSII) and had previously reported a BBD diagnosis (either prior to study entry or after study baseline); were diagnosed with breast cancer a median of 7.67 years after BBD diagnoses (interquartile range 4.33 to 11.75 years); and were excluded if the time between BBD and breast cancer diagnoses was less than six months or if there was evidence of invasive or in situ carcinoma during centralized histopathological review of the BBD lesion. Tumor estrogen receptor (ER) status was obtained from centralized review of breast tissue microarrays ${ }^{43}$. If centralized review data was unavailable then ER status was retrieved from pathology reports. Controls were women diagnosed with BBD who did not develop breast cancer. Cases and controls were matched 1:4 on year of BBD diagnosis, age at breast cancer diagnosis (index date for controls), and years between BBD and breast cancer diagnosis (or index date). A total of 3795 slides were digitized at $20 \times(n=213)$ or $40 \times$ magnification $(n=3582)$. Each woman contributed between one to four WSIs (median WSIs $n=3$ ). 
The histological type of the BBD lesion was determined by central pathology review. Participant body mass index (BMI), age at menarche, parity, age at first birth, breastfeeding history, and menopausal status were obtained from questionnaires of the participants closest to but prior to BBD biopsy. The average body sizes at ages 5 and 10 reported by using a ninelevel pictogram (Level 1 as leanest) ${ }^{34}$. Birth index, a surrogate metric that reflects the timing and spacing of births, was calculated as previously described ${ }^{44}$. A higher birth index indicates a higher number of births occurring at earlier ages.

Application Phase: Applying our networks to WSIs from the BBD NCC study participants

Figure 1 shows an overview of our BBD image analysis pipeline. For each WSI, binary tissue masks were generated using simple color thresholding at a magnification level of $0.25 \times$ (Figure 1B). After tissue-containing areas were located, each WSI was split into patches of size 2048 x 2048 pixels. Each patch was extracted at a 10x magnification level, and tissue segmentation and nuclei detection were performed using the networks established in the development phase (Figure $1 \mathrm{C}$ ). Each patch resulted as a segmentation map with each pixel classified as epithelium, fibrous stroma, fat, or background.

Application Phase: Extracting percentages of each tissue region

Each tissue region was expressed as a percentage of the total amount of tissue analyzed for each woman. Pixels classified as epithelium, fibrous stroma, or fat were individually summed across patches from a single WSI, combined across WSIs pertaining to each woman, and divided by the total number of pixels detected across all tissue regions. 
Application Phase: Extracting morphometric features

Morphology, texture, and graph-based spatial features (i.e., computer-derived morphometric features; $n=619)$ were extracted using the WSIs in conjunction with the automated tissue segmentation and nuclei detection results (Figure 1D). Since fat regions were mostly empty white spaces, fat and fibrous stroma regions were combined as stroma for feature extraction. For each tissue region, 25 morphological features summarized the areas, perimeters, spatial densities, and red green blue color distributions of detected nuclei; 161 features summarized various geometric properties (e.g. eccentricity, convex area) and image moments ${ }^{45}$; 113 Haralick texture ${ }^{46}$ and local binary pattern ${ }^{47}$ features were calculated using the grayscale co-occurrence matrices. The 598 features detailed above were computed at the patch level and then aggregated across all patches belonging to a single WSI. At the WSI level, an additional 21 graph-based spatial features (i.e., Voronoi diagrams ${ }^{48}$ and Delaunay triangulations ${ }^{49}$ ) were computed directly to characterize the holistic spatial distribution of the epithelial regions.

For women with more than one WSI, the value for each feature was further summarized using the median calculated across all her WSIs. Four features were subsequently excluded due to missing values-percentage area and the standard deviation of major axis length for both epithelial and stroma areas. Thus, a total of 615 features were analyzed in this study. 
The 1425 study participants were split into a training:test ratio of 60:40. The sampling package in R ensured the test set of 570 women (40\%) was representative of the training set by selecting women based on outcome (case/control) and BBD histopathological subtypes. A morphometric signature associated with breast cancer was constructed using a training set of 855 women (60\%), $615 z$-scored morphometric features, and elastic net regularized regression model with 1000 iterations and 10 -fold cross validation (glmnet package in $\mathrm{R}^{50}$ ). The morphometric signature was subjected to bootstrapping (1000 iterations) to obtain $95 \%$ confidence intervals for feature coefficient estimates. Features whose coefficients with $95 \%$ confidence intervals crossing zero were dropped from the signature. A signature score for each woman in the test set was computed by summing the products of the final selected features' coefficients and z-scored values.

Application Phase: Statistical Analysis

Preliminary assessments using Wilcoxon rank sum test evaluated if there was any difference in amount of epithelial, fibrous stroma, and fat tissues between cases and controls. Kruskal-Wallis test was used to determine if each tissue region was different between case/control statuses, stratified by BBD histological subtypes. Significant Kruskal-Wallis tests were further evaluated using Dunn's post hoc tests with Benjamini-Hochberg multiple testing method to obtain adjusted $p$-values (FSA R package $0.8 .30^{51}$ ). Only meaningful significant (adjusted $p<0.05$ ) comparisons within cases, controls, and between case and controls were reported. 
The associations between breast cancer risk factors and tissue composition (natural logtransformed) among controls were assessed using analysis of covariance (ANCOVA) adjusting for age at BBD biopsy (emmeans R package version 1.4.4 ${ }^{52}$ ). Each tissue region was categorized into quartiles as defined by the distribution among controls. Unconditional logistic regression models accounting for the matching factors to estimate odd ratios (ORs) and $95 \%$ confidence intervals $(\mathrm{Cl})$ was used to determine the relationship between each tissue region (in quartiles) and breast cancer risk (Figure 1E). Unconditional logistic regression models were used because of incomplete matched case-control sets-lack of pathology records and/or slides for all selected cases and controls. Model 1 adjusted for matching factors (year of BBD biopsy, age at index date, time between BBD biopsy and index date); model 2 adjusted for matching factors and BBD histological subtypes; and model 3 adjusted for matching factors, BBD histological subtypes, parity, menopausal status, and BMI. Analyses were also conducted by stratifying the women according to BBD histological subtype, parity, menopausal status, or BMI. Polytomous logistic regression models assessed the association between each tissue region and risk of breast cancer defined by tumor ER expression.

The morphometric signature scores were winsorized to $96 \%$ where scores below the second percentile were set to the score at the second percentile and scores above the 98th percentile were set to the score at the 98th percentile. The association of this score and breast cancer outcome in the held-out test set was similarly evaluated using unconditional logistic 
medRxiv preprint doi: https://doi.org/10.1101/2020.09.02.20186643; this version posted September 3, 2020. The copyright holder for this preprint

(which was not certified by peer review) is the author/funder, who has granted medRxiv a license to display the preprint in perpetuity.

It is made available under a CC-BY-NC-ND 4.0 International license.

regression models. The level of significance used for all statistical tests was $p<0.05$. All statistical analyses were performed using $R$. 


\section{Results}

Tissue segmentation and nuclei detection networks performance (development phase)

The precision, recall, and Dice similarity coefficient of the tissue segmentation network were $0.79,0.71$, and 0.75 for epithelium; $0.83,0.88$, and 0.85 for fibrous stroma; $0.85,0.67$, and 0.75 for fat; and $0.84,0.92$, and 0.88 for background. The cell nuclei detection network detected nuclei with a precision of 0.93 , recall of 0.71 , and Dice similarity coefficient of 0.81 . An example of an original image, ground truth annotation, and automated segmentation or detection for each network is presented in Figure 2.

\section{BBD NCC study participants}

Table 1 displays the matching factors and BBD histopathological subtypes of the 293 breast cancer cases and 1132 controls. The majority of the women were diagnosed with proliferative breast disease without atypia. The average age at breast cancer diagnosis among

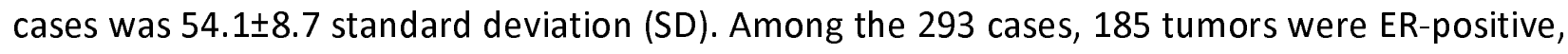
51 were ER-negative, and 57 were unknown.

\section{Preliminary assessment of breast tissue composition}

Crude Wilcoxon tests compared cases and controls within each tissue region (Figure 3A). Cases have significantly more epithelium $(p<0.001)$ and suggestively more fibrous stroma $(p=0.07)$ than controls. Controls have significantly more fat $(p<0.001)$ than cases. When stratified by BBD histological subtypes, there were significant differences among cases or 
controls, or between cases and controls for each tissue region (epithelium $p<0.001$, fibrous stroma $p=0.02$, fat $p<0.001$; Kruskal-Wallis tests). Dunn's post-hoc analyses with BenjaminiHochberg multiple testing adjustment revealed that among cases, those with proliferative without atypia subtype had significantly more epithelium compared to those with nonproliferative (adjusted $p=0.03$ ) or proliferative with atypia subtype (adjusted $p=0.004$; Figure 3B). Among controls, those with non-proliferative subtype had significantly less epithelium than those with proliferative without atypia (adjusted $p<0.001$ ) or proliferative with atypia subtypes (adjusted $p=0.009$; Figure 3B). When comparing between cases and controls, cases with nonproliferative or proliferative without atypia subtype have significantly more epithelium compared to controls (adjusted $p=0.02$ and 0.002, respectively; Figure 3B).

There was no pairwise difference in the amount of fibrous stroma among cases or controls, or between cases and controls within each BBD histological subtype in crude analyses (adjusted $p>0.05$ for all comparisons; Figure $3 \mathrm{C}$ ). Cases with proliferative without atypia subtype have less fat than cases with non-proliferative (adjusted $p=0.05$ ) or proliferative with atypia subtypes (adjusted $p=0.002$; Figure 3D). Likewise, controls with proliferative without atypia subtype have less fat than controls with non-proliferative (adjusted $p=0.001$ ) or proliferative with atypia subtypes (adjusted $p=0.09$; Figure 3D). Within the proliferative without atypia subtype, cases have significantly less fat compared to controls (adjusted $p<0.001$; Figure 3D). 
Table 2 displays the age-adjusted means $(95 \% \mathrm{CI})$ and the ANCOVA $p$-values of the associations between BBD histological subtypes, body size, and reproductive breast cancer risk factors and the tissue composition among the controls. The relationship between BBD histological subtypes and percentages of epithelium and fat among controls remained significant in age-adjusted analyses (both $p<0.001$ ). In contrast to crude analyses, controls with proliferative subtypes have significantly more fibrous stroma compared to controls with nonproliferative subtype after adjusting for age $(p<0.001)$.

Women with a larger childhood body size (Levels $1.5-2$ and $\geq 2.5$ ) had less fibrous stroma $(p=0.048)$ but suggestively more fat $(p=0.09)$ compared to women with body sizes of 1 or 1.5 to 2 at ages 5-10 years. Breast tissues of women with BMI $\geq 30$ at the time of BBD biopsy had lower amount of fibrous stroma $(p<0.001)$ but higher amount of fat $(p<0.001)$ compared to women with lower BMI.

Parous women had more epithelium and fat, and less fibrous stroma compared to nulliparous women (all $p<0.05$; Table 2). When parous women were further subdivided, women who had $\geq 2$ births (multiparous) had more epithelium and fat, and less fibrous stroma than women who had one birth (primiparous) or nulliparous women $(p<0.05)$. When parous women were subdivided into women whose last birth was $<20$ years or $\geq 20$ years prior to BBD diagnosis, women who had their last birth $<20$ years had more epithelium and fat compared to nulliparous women and women who had their last birth $\geq 20$ years prior to BBD diagnosis $(p<0.05)$. Post-menopausal women had less epithelium $(p=0.001)$ and fibrous stroma $(p=0.004)$ 
compared to pre-menopausal women (Table 2). The age of menarche positively correlated with the amount of fibrous stroma $(p=0.01)$. Age at first birth, birth index, and breastfeeding were not associated with breast tissue composition.

\section{Tissue composition and breast cancer risk}

A higher proportion of epithelium was significantly associated with subsequent breast cancer risk when accounting for matching factors (OR=1.53, 95\% Cl 1.04-2.27 comparing highest and lowest quartiles; $p$-trend=0.02). Upon additional adjustment for BBD subtype, parity, menopausal status, and BMI, the association modestly attenuated but remained significantly elevated (OR=1.39, 95\% $\mathrm{Cl} 0.91-2.14$ comparing highest and lowest quartiles; $p$ trend=0.047; Table 3). Neither the amount of fibrous stroma nor fat was associated with breast cancer risk in all three models (all $p$-trend>0.05; Table 3).

Additional analyses were conducted, stratifying by BBD, parity, menopausal status, or BMI. Within the BBD proliferative without atypia subtype, women with epithelium in the fourth quartile had higher breast cancer risk compared to women in the first quartile (adjusted OR=1.92, $95 \% \mathrm{Cl} 1.11-3.40 ; p$-trend=0.01; Supplementary Table 2). Polytomous logistic regression models assessed the association between tissue regions and breast cancer risk defined by tumor ER expression, and in general demonstrated no heterogeneity. Fat was associated with lower breast cancer risk among ER-positive women in the crude model 1 (second versus first tertile: $\mathrm{OR}=0.62,95 \% \mathrm{Cl} 0.42-0.92$; third versus first tertile: $\mathrm{OR}=0.62,95 \% \mathrm{Cl}$ 
To understand the substitution effects, further analyses were conducted using each tissue region as a continuous variable per $10 \%$ change and with two of the three tissue regions in the model (Supplementary Table 4). Similar to Table 3 where tissue regions were analyzed using quartiles, the association between epithelium as a continuous variable and breast cancer risk remained the strongest. Every $10 \%$ increase in the amount of epithelium was significantly associated with increased breast cancer risk in fully adjusted models, irrespective of whether it is substituted for fibrous stroma (adjusted $\mathrm{OR}=1.30,95 \% \mathrm{Cl} 1.05-1.61$ ) or fat tissue (adjusted OR=1.26, 95\% Cl 1.03-1.54; Supplementary Table 4).

Morphometric signature associated with subsequent breast cancer risk

The morphometric signature developed using elastic net regularized regression consisted of four features in the epithelium: the kurtosis of delaunay edge length (fourth moment of a graph-based feature which measures the spatial density (interconnectedness) of nuclei found in each epithelial region), the kurtosis of euler number (fourth moment of a topological feature which measures the number of holes in an epithelial region), mean perimeter of epithelial regions, and the minimum blue channel pixel value of nuclei found in epithelial regions. The area under the receiver operator curve (AUC ROC) of the model was 0.61 (optimal $\lambda=0.08$ ). When evaluated on the test set of 570 women, the AUC ROC was 0.51. Due to the poor AUC ROC of the test set, the association of the signature score with breast cancer was not further evaluated. 


\section{Discussion}

The identification of new biomarkers may improve breast cancer risk prediction. We developed a deep-learning based computational pathology method to segment BBD histopathological images into epithelial, fibrous stroma, and fat regions; calculate tissue composition; and extract morphometric features. We applied our method to the BBD breast cancer NCC study within the NHS/NHSII to determine the association of the tissue composition with breast cancer risk factors and breast cancer risk. Among controls, BBD subtypes, parity, and number of births were significantly associated with all three tissue regions; select tissue regions were associated with body size at ages 5-10 years, BMI, age of menarche, and menopausal status. Women whose breast tissues had higher percentages of epithelium had significantly increased risk of breast cancer compared to women with lower percentages, especially among women with proliferative without atypia subtype of BBD. We also extracted computer-derived morphometric features from each tissue region but were unable to construct a robust breast cancer morphometric signature. Our study showed that the percentage of epithelium may be utilized as a potential biomarker of breast cancer risk.

BBD and breast cancer originate from TDLUs. The epithelium captured by our computational method was all-encompassing-normal TDLUs, TDLUs with proliferative or metaplastic changes, and various types of BBD lesions. Hence, our study was the first to demonstrate a direct quantitative relationship between the percentage of epithelium and subsequent breast cancer risk in women diagnosed with BBD. Our findings also supported the 
long-held hypothesis that elevated cellular mass increases risk of cancer ${ }^{53}$. Some lesion types within the proliferative without atypia subtype are highly cellular. For example, adenosis is characterized by increased number of enlarged lobules while radial scar is characterized by a central area of fibroelastotic change from which epithelial structures with or without associated hyperplasia or metaplasia radiate. This explained why when stratified by BBD subtype, the association of the percentage of epithelium and breast cancer risk remained significant among women within the proliferative without atypia of BBD.

The associations of age-adjusted breast tissue composition and breast cancer risk factors among controls provided histopathological evidence to support epidemiological studies, mainly by demonstrating the link between breast tissue cellularity and cancer risk ${ }^{53}$. Our work suggests that risk factors have different influences on the percentages of epithelium and fibrous stroma. Gertig et al. evaluated the proportion of epithelium and fibrous stroma in 300 BBD women who did not develop breast cancer ${ }^{54}$. We and Gertig et al. showed that breast tissues associated with the non-proliferative subtype of BBD were less cellular (i.e., lower epithelium and fibrous stroma but higher fat percentages) than proliferative with or without atypia subtypes, thus partly explaining why women with the non-proliferative subtype have lower breast cancer risk compared to proliferative subtypes ${ }^{33,55-58}$.

Adiposity during childhood or in young adults is inversely associated with breast cancer risk ${ }^{59-61}$. Body adiposity is correlated with the amount of fat tissue in the breast when evaluated using percentage mammographic density (i.e., proportion of dense (epithelium and 
fibrous stroma) to non-dense tissues (fat)) ${ }^{62,63}$. As such, percentage mammographic density is also inversely associated with breast cancer risk. In 153 normal breast tissue samples obtained via core-biopsy, Gabrielson et al. observed significant inverse associations of BMI with percentages of epithelium and stroma ${ }^{64}$. However, our study and the study by Gertig et $a l^{54}$ were conducted using more participants and only observed a significant inverse association between BMI and proportion of stroma. Nevertheless, these three studies provided histologic evidence to partially explain the differential breast cancer risk by adiposity-breast tissues of women with a larger childhood body size or younger women with BMI $\geq 30$ have lower overall cellularity (i.e., epithelium and fibrous stroma) and thus are less dense compared to women with a leaner childhood body size or women with lower BMI, respectively.

Parity had the strongest influence on breast tissue composition among the reproductive risk factors investigated in our study. Gertig et al. and Gabrielson et al. observed more epithelium and less fibrous stroma in parous women compared to nulliparous women ${ }^{54,64}$. Our observation of higher percentages of epithelium and fat, and lower fibrous stroma percentages in multiparous women who had a live birth within the last 20 years was similar to other studies that observed less TDLU involution in parous versus nulliparous women ${ }^{24,65}$; supported epidemiological reports of increased breast cancer risk in parous women who had a live birth within the last 5 to 24 years compared to nulliparous women ${ }^{66}$; and highlighted the extensive stroma remodeling in mammary glands during pregnancy to accommodate expanding epithelium ${ }^{67}$. The correlation between age of menarche and proportion of stroma reported by us and others ${ }^{54,64}$ is in line with higher percent breast density in young women who had later 
ages of menarche ${ }^{68}$. The null associations between age of first birth and length of breastfeeding with breast tissue composition agreed with Gertig et al. ${ }^{54}$ while Gabrielson et al. ${ }^{64}$ found an association between percentage of epithelium and length of breastfeeding $(\beta=0.03 \pm$ $0.01, p=0.02)$, but not percentage of stroma $(\beta=0.43 \pm 0.26, p=0.10)$. Using a different computational pathology method that specifically measures normal TDLUs, we also did not find an association between length of breastfeeding and TDLU involution ${ }^{24}$. Older women have less dense breasts than younger women, with the greatest change in density occurring during the menopause years ${ }^{69}$. Indeed, we and Gertig et al. ${ }^{54}$ reported that post-menopausal women had less epithelium and fibrous stroma compared to pre-menopausal women. However, this was not observed by Gabrielson et al. ${ }^{64}$, possibly due to low power.

Computer-derived morphometric signatures have shown potential as prognostic or diagnostic biomarkers ${ }^{15,16,70}$. We did not identify a breast cancer morphometric signature from BBD WSIs. Morphometric feature data are typically noisy. In an effort to reduce signal noise, we attempted unsuccessfully to create a breast cancer signature within each BBD histopathological subtype due to low power. There were more features than the number of samples. Extracting and combining morphometric features from different types of epithelium may have excessively diluted meaningful signals. Using the median metric ${ }^{15}$, a common method of aggregating morphometric features across all WSIs, may not optimal for this dataset. There is no gold standard method for feature aggregation and this remains an active area of research by medical image analysis groups. Future work can include improving methods for morphometric feature 
aggregation or create specific breast cancer morphometric signatures for each type of BBD lesion.

The strengths of our study include the application of a computer pathology method to assess breast tissue composition in a large nested case-control study with rich data on breast cancer risk factors ${ }^{3,4,26,27,32,34}$. BBD samples underwent centralized pathology review and breast cancer cases were confirmed through review of medical records. Some limitations of our study include being underpowered to evaluate the association of breast composition and ER negative breast cancer, breast cancer molecular subtypes ${ }^{71,72}$, or mammographic density ${ }^{73,74}$ as mammogram data were only available for 105 women $(7.8 \%)$ in this study. Our findings were also limited to White women, the predominant race of the NHS/NHSII participants. Dysfunctional epithelial-stroma interactions in the breast have been implicated in breast carcinogenesis ${ }^{75}$, however, our current study was not designed to investigate epitheliumstroma interactions.

In conclusion, we found that BBD histopathological subtypes, anthropometric, and selective reproductive risk factors were associated with breast tissue composition. Higher percentages of epithelium were associated with increased risk of breast cancer, specifically among women with the proliferative without atypia subtype of BBD. No morphometric signature was associated with subsequent breast cancer. Future work can include incorporation of the percentage of epithelium into risk assessment models as well as explore end-to-end deep learning breast cancer prediction models. 
medRxiv preprint doi: https://doi.org/10.1101/2020.09.02.20186643; this version posted September 3, 2020. The copyright holder for this preprint

(which was not certified by peer review) is the author/funder, who has granted medRxiv a license to display the preprint in perpetuity. It is made available under a CC-BY-NC-ND 4.0 International license.

\section{Acknowledgements}

We thank the participants and staff of the Nurses' Health Study and Nurses' Health Study II for their valuable contributions as well as the following state cancer registries for their help: $A L, A Z$, $A R, C A, C O, C T, D E, F L, G A, I D, I L, I N, I A, K Y, L A, M E, M D, M A, M I, N E, N H, N J, N Y, N C, N D, O H$, OK, OR, PA, RI, SC, TN, TX, VA, WA, WY. The authors assume full responsibility for analyses and interpretation of these data. 


\section{References}

1. DeSantis CE, Ma J, Gaudet MM, et al. Breast cancer statistics, 2019. CA Cancer J Clin. 2019;69(6):438-451. doi:10.3322/caac.21583

2. Kensler KH, Beca F, Baker GM, et al. Androgen receptor expression in normal breast tissue and subsequent breast cancer risk. npj Breast Cancer. 2018;4(1):33. PMCID: PMC6155011. doi:10.1038/s41523-018-0085-3

3. Aroner SA, Collins LC, Connolly JL, et al. Radial scars and subsequent breast cancer risk: Results from the Nurses' Health Studies. Breast Cancer Res Treat. 2013;139(1):277-85. PMCID: PMC3689547. doi:10.1007/s10549-013-2535-9

4. Collins LC, Aroner SA, Connolly JL, Colditz GA, Schnitt SJ, Tamimi RM. Breast cancer risk by extent and type of atypical hyperplasia: An update from the Nurses' Health Studies. Cancer. 2016;122(4):515-20. PMCID: PMC4742394. doi:10.1002/cncr.29775

5. Zhang H, Ahearn T, Lecarpentier J, Barnes D, Beesley J, Qi G, Jiang X, O'Mara TA, Zhao N, Bolla MK, Dunning AM, Dennis J, Wang Q, Ful ZA, Aittomäki K, Andrulis IL, Anton-Culver $H$, Arndt V, Aronson KJ, Arun BK, Auer PL, Azzollini J, Barrowdale D, Becher H, Beckmann MW, Behrens S, Benitez J, G-CM. Genome-wide association study identifies 32 novel breast cancer susceptibility loci from overall and subtype-specific analyses. Nat Genet. 2020;52(6):572-581.

6. $\quad$ Wang J, Eliassen AH, Spiegelman D, Willett WC, Hankinson SE. Plasma free 25hydroxyvitamin $D$, vitamin D binding protein, and risk of breast cancer in the Nurses' Health Study II. Cancer Causes Control. 2014;25(7):819-827. doi:10.1007/s10552-0140383-5

7. Kotsopoulos J, McGee EE, Lozano-Esparza S, et al. Premenopausal Plasma Osteoprotegerin and Breast Cancer Risk: A Case-Control Analysis Nested within the Nurses' Health Study II. Cancer Epidemiol Biomarkers Prev. 2020;29(6):1264-1270. doi:10.1158/1055-9965.epi-19-1154

8. Tice J, Cummings S, Smith-Bindman R, Ichikawa L, Barlow W, Kerlikowske K. Using clinical factors and mammographic breast density to estimate breast cancer risk: Development and validation of a new predictive model. Ann Intern Med. 2008;148(5):337-347.

9. Tice JA, Miglioretti DL, Li C-S, Vachon CM, Gard CC, Kerlikowske K. Breast density and benign breast disease: Risk assessment to identify women at high risk of breast cancer. $J$ Clin Oncol. 2015;33(28):3137-43. PMCID: PMC4582144. doi:10.1200/JC0.2015.60.8869

10. Rice MS, Tworoger SS, Hankinson SE, et al. Breast cancer risk prediction: an update to the Rosner-Colditz breast cancer incidence model. Breast Cancer Res Treat.

2017;166(1):227-240. doi:10.1007/s10549-017-4391-5

11. Zhang $X$, Rice $M$, Tworoger SS, et al. Addition of a polygenic risk score, mammographic density, and endogenous hormones to existing breast cancer risk prediction models: A nested case-control study. PLoS Med. 2018;15(9):e1002644.

doi:10.1371/journal.pmed.1002644

12. Pastor-Barriuso R, Ascunce N, Ederra M, et al. Recalibration of the Gail model for predicting invasive breast cancer risk in Spanish women: A population-based cohort study. Breast Cancer Res Treat. 2013;138(1):249-259. doi:10.1007/s10549-013-2428-y

13. Veta M, Heng YJ, Stathonikos N, et al. Predicting breast tumor proliferation from whole- 
slide images: The TUPAC16 challenge. Presented at the: 2019.

doi:10.1016/j.media.2019.02.012

14. Bejnordi BE, Veta M, van Diest PJ, et al. Diagnostic assessment of deep learning algorithms for detection of lymph node metastases in women with breast cancer. JAMA. 2017;318(22):2199-2210. PMCID: PMC5820737. doi:10.1001/jama.2017.14585

15. Tian K, Rubadue CA, Lin DI, et al. Automated clear cell renal carcinoma grade classification with prognostic significance. PLoS One. 2019;14(10):e0222641. doi:10.1371/journal.pone.0222641

16. Beck AH, Sangoi AR, Leung $S$, et al. Systematic analysis of breast cancer morphology uncovers stromal features associated with survival. Sci Trans/ Med. 2011;3(108):108ra113. doi:10.1126/scitranslmed.3002564

17. Tabesh A, Teverovskiy M, Pang HY, et al. Multifeature prostate cancer diagnosis and gleason grading of histological images. IEEE Trans Med Imaging. 2007;26(10):1366-1378. doi:10.1109/TMI.2007.898536

18. Yu K-H, Zhang C, Berry GJ, et al. Predicting non-small cell lung cancer prognosis by fully automated microscopic pathology image features. Nat Commun. 2016;7:12474. doi:10.1038/ncomms12474

19. Ehteshami Bejnordi B, Lin J, Glass B, et al. Deep learning-based assessment of tumorassociated stroma for diagnosing breast cancer in histopathology images. In: Proceedings - International Symposium on Biomedical Imaging. ; 2017:929-932. doi:10.1109/ISBI.2017.7950668

20. Xu J, Luo X, Wang G, Gilmore H, Madabhushi A. A Deep Convolutional Neural Network for segmenting and classifying epithelial and stromal regions in histopathological images. Neurocomputing. 2016;191:214-223. doi:10.1016/j.neucom.2016.01.034

21. Dong F, Irshad H, Oh EY, et al. Computational pathology to discriminate benign from malignant intraductal proliferations of the breast. PLoS One. 2014;9(12):e114885. doi:10.1371/journal.pone.0114885

22. Wetstein SC, Onken AM, Baker GM, et al. Detection of acini in histopathology slides: towards automated prediction of breast cancer risk. In: Proc. SPIE 10956, Medical Imaging 2019: Digital Pathology. Vol 10956. ; 2019:109560Q. doi:10.1117/12.2511408

23. Wetstein SC, Onken AM, Luffman C, et al. Deep learning assessment of breast terminal duct lobular unit involution?: towards automated prediction of breast cancer risk. PLOS One. 2020;15(4):e0231653.

24. Kensler KH, Liu EZ, Wetstein SC, et al. The Association of Automated Quantitative Measures of Terminal Duct Lobular Unit Involution and Breast Cancer Risk. Cancer Epidemiol Biomarkers Prev. 2020:In Press.

25. Colditz GA, Hankinson SE. The Nurses' Health Study: lifestyle and health among women. Nat Rev Cancer. 2005;5(5):388-396. doi:10.1038/nrc1608

26. Collins LC, Baer HJ, Tamimi RM, Connolly JL, Colditz GA, Schnitt SJ. The influence of family history on breast cancer risk in women with biopsy-confirmed benign breast disease: results from the Nurses' Health Study. Cancer. 2006;107(6):1240-1247. doi:10.1002/cncr.22136

27. Tamimi RM, Byrne C, Baer HJ, et al. Benign breast disease, recent alcohol consumption, and risk of breast cancer: a nested case-control study. Breast Cancer Res. 
2005;7(4):R555-62. PMCID: PMC1175067. doi:10.1186/bcr1039

28. Su X, Colditz GA, Willett WC, et al. Genetic variation and circulating levels of IGF-I and IGFBP-3 in relation to risk of proliferative benign breast disease. Int J Cancer. 2010;126(1):180-190. doi:10.1002/ijc.24674

29. Baer HJ, Schnitt SJ, Connolly JL, et al. Early life factors and incidence of proliferative benign breast disease. Cancer Epidemiol Biomarkers Prev. 2005;14(12):2889-2897. doi:10.1158/1055-9965.EPI-05-0525

30. Farland L V., Tamimi RM, Eliassen AH, et al. A prospective study of endometriosis and risk of benign breast disease. Breast Cancer Res Treat. 2016;159(3):545-52. PMCID: PMC5310266. doi:10.1007/s10549-016-3957-y

31. Jung MM, Colditz GA, Collins LC, Schnitt SJ, Connolly JL, Tamimi RM. Lifetime physical activity and the incidence of proliferative benign breast disease. Cancer Causes Control. 2011;22(9):1297-1305. doi:10.1007/s10552-011-9803-y

32. Baer HJ, Collins LC, Connolly JL, Colditz GA, Schnitt SJ, Tamimi RM. Lobule type and subsequent breast cancer risk: Results from the nurses' health studies. Cancer. 2009;115(7):1404-11. PMCID: PMC2661011. doi:10.1002/cncr.24167

33. Collins LC, Baer HJ, Tamimi RM, Connolly JL, Colditz GA, Schnitt SJ. Magnitude and laterality of breast cancer risk according to histologic type of atypical hyperplasia: Results from the nurses' health study. Cancer. 2007;109(2):180-187. doi:10.1002/cncr.22408

34. Oh $\mathrm{H}$, Eliassen $\mathrm{AH}$, Wang $\mathrm{M}$, et al. Expression of estrogen receptor, progesterone receptor, and Ki67 in normal breast tissue in relation to subsequent risk of breast cancer. npj Breast Cancer. 2016;2(1):16032. doi:10.1038/npjbcancer.2016.32

35. Beca F, Kensler K, Glass B, Schnitt SJ, Tamimi RM, Beck AH. EZH2 protein expression in normal breast epithelium and risk of breast cancer: Results from the Nurses' Health Studies. Breast Cancer Res. 2017;19(1):R32. doi:10.1186/s13058-017-0817-6

36. Simonyan K, Zisserman A. Very deep convolutional networks for large-scale image recognition. 3rd International Conference on Learning Representations, ICLR 2015 Conference Track Proceedings. http://arxiv.org/abs/1409.1556. Published September 4, 2015. Accessed May 1, 2018.

37. Ronneberger O, Fischer P, Brox T. U-Net: Convolutional Networks for Biomedical Image Segmentation. http://arxiv.org/abs/1505.04597. Published May 18, 2015. Accessed May $1,2018$.

38. Long J, Shelhamer E, Darrell T. Fully convolutional networks for semantic segmentation. In: 2015 IEEE Conference on Computer Vision and Pattern Recognition (CVPR). IEEE; 2015:3431-3440. doi:10.1109/CVPR.2015.7298965

39. Stark F, Triebel R, Cremers D. CAPTCHA Recognition with Active Deep Learning. 37th Ger Conf Pattern Recognit. 2015:94.

40. Scheffer T, Decomain C, Wrobel S. Active hidden markov models for information extraction. In: Lecture Notes in Computer Science (Including Subseries Lecture Notes in Artificial Intelligence and Lecture Notes in Bioinformatics). Vol 2189. ; 2001:309-318. doi:10.1007/3-540-44816-0_31

41. Kingma DP, Ba JL. Adam: A method for stochastic optimization. In: 3rd International Conference on Learning Representations, ICLR 2015 - Conference Track Proceedings. ; 2015. 
42. Kumar N, Verma R, Sharma S, Bhargava S, Vahadane A, Sethi A. A dataset and a technique for generalized nuclear segmentation for computational pathology. IEEE Trans Med Imaging. 2017;36(7):1550-1560. doi:10.1109/TMI.2017.2677499

43. Tamimi RM, Baer HJ, Marotti J, et al. Comparison of molecular phenotypes of ductal carcinoma in situ and invasive breast cancer. Breast Cancer Res. 2008;10(4):R67. doi:10.1186/bcr2128

44. Sisti JS, Collins LC, Beck AH, Tamimi RM, Rosner BA, Eliassen AH. Reproductive risk factors in relation to molecular subtypes of breast cancer: Results from the nurses' health studies. Int J Cancer. 2016;138(10):2346-2356. doi:10.1002/ijc.29968

45. Van Der Walt S, Schönberger JL, Nunez-Iglesias J, et al. Scikit-image: Image processing in python. PeerJ. 2014;2014(2):e453. doi:10.7717/peerj.453

46. Haralick RM, Shanmugam K, Dinstein I. Textural features for image classification. IEEE Trans Syst Man Cybern. 1973;SMC-3(6):610-621. doi:10.1109/TSMC.1973.4309314

47. Doshi N, Schaefer G. A comparative analysis of local binary pattern texture classification. In: 2012 IEEE Visual Communications and Image Processing, VCIP 2012. San Diego; 2012:1-6. doi:10.1109/VCIP.2012.6410773

48. Martínez A, Martínez J, Pérez H, Quirós R. Image processing using Voronoi diagrams. In: Proceedings of the 2007 International Conference on Image Processing, Computer Vision, and Pattern Recognition, IPCV 2007. Las Vegas, Nevada; 2007:485-491.

49. Kohout J. On digital image representation by the delaunay triangulation. In: Lecture Notes in Computer Science (Including Subseries Lecture Notes in Artificial Intelligence and Lecture Notes in Bioinformatics). Vol 4872 LNCS. ; 2007:826-840. doi:10.1007/978-3-54077129-6_70

50. Friedman J, Hastie T, Tibshirani R. Regularization paths for generalized linear models via coordinate descent. J Stat Softw. 2010;33(1):1-22. PMCID: PMC2929880.

doi:10.1359/JBMR.0301229

51. Ogle D, Powell W, Dinno A. FSA: Simple Fisheries Stock Assessment Methods. R package. https://cran.r-project.org/package=FSA. Published 2020.

52. Lenth R, Singmann H, Love J, Buerkner P, Herve M. Estimated Marginal Means, aka LeastSquares Means. R package. https://cran.r-project.org/package=emmeans. Published 2020.

53. Preston-Martin S, Pike MC, Ross RK, Henderson BE, Jones PA. Increased Cell Division as a Cause of Human Cancer. Cancer Res. 1990;50(23):7415-7421.

54. Gertig DM, Stillman IE, Byrne C, et al. Association of age and reproductive factors with benign breast tissue composition. Cancer Epidemiol Biomarkers Prev. 1999;8(10):873-879.

55. Dupont WD, Page DL. Risk factors for breast cancer in women with proliferative breast disease. N Engl J Med. 1985;312(3):146-151. doi:10.1056/NEJM198501173120303

56. Hartmann LC, Sellers TA, Frost MH, et al. Benign breast disease and the risk of breast cancer. N Engl J Med. 2005;353(3):229-237. doi:10.1056/NEJMoa044383

57. Dyrstad SW, Yan Y, Fowler AM, Colditz GA. Breast cancer risk associated with benign breast disease: systematic review and meta-analysis. Breast Cancer Res Treat. 2015;149(3):569-575. doi:10.1007/s10549-014-3254-6

58. Menes TS, Kerlikowske K, Lange J, Jaffer S, Rosenberg R, Miglioretti DL. Subsequent Breast Cancer Risk Following Diagnosis of Atypical Ductal Hyperplasia on Needle Biopsy. 
JAMA Oncol. 2017;3(1):36-41. doi:10.1001/jamaoncol.2016.3022

59. Schoemaker MJ, Nichols HB, Wright LB, et al. Association of Body Mass Index and Age with Subsequent Breast Cancer Risk in Premenopausal Women. JAMA Oncol. 2018;4(11):e181771. doi:10.1001/jamaoncol.2018.1771

60. Baer HJ, Colditz GA, Rosner B, et al. Body fatness during childhood and adolescence and incidence of breast cancer in premenopausal women: A prospective cohort study. Breast Cancer Res. 2005;7(3). doi:10.1186/bcr998

61. Baer HJ, Tworoger SS, Hankinson SE, Willett WC. Body fatness at young ages and risk of breast cancer throughout life. Am J Epidemiol. 2010;171(11):1183-1194. doi:10.1093/aje/kwq045

62. Oh H, Rice MS, Warner ET, et al. Early-life and adult anthropometrics in relation to mammographic image intensity variation in the nurses' health studies. Cancer Epidemiol Biomarkers Prev. 2020;29(2):343-351. doi:10.1158/1055-9965.EPI-19-0832

63. Byrne C, Schairer C, Wolfe J, et al. Mammographic features and breast cancer risk: Effects with time, age, and menopause status. J Nat/ Cancer Inst. 1995;87(21):1622-1629. doi:10.1093/jnci/87.21.1622

64. Gabrielson M, Chiesa F, Behmer C, Rönnow K, Czene K, Hall P. Association of reproductive history with breast tissue characteristics and receptor status in the normal breast. Breast Cancer Res Treat. 2018;170(3):487-497. doi:10.1007/s10549-018-4768-0

65. Russo J, Hu YF, Yang X, Russo IH. Developmental, cellular, and molecular basis of human breast cancer. J Natl Cancer Inst Monogr. 2000;(27):17-37. doi:10.1093/oxfordjournals.jncimonographs.a024241

66. Nichols HB, Schoemaker MJ, Cai J, et al. Breast cancer risk after recent childbirth: A pooled analysis of 15 prospective studies. Ann Intern Med. 2019;170(1):22-30. doi:10.7326/M18-1323

67. McCready J, Arendt LM, Rudnick JA, Kuperwasser C. The contribution of dynamic stromal remodeling during mammary development to breast carcinogenesis. Breast Cancer Res. 2010;12(3):205. doi:10.1186/bcr2578

68. Houghton LC, Jung S, Troisi R, et al. Pubertal timing and breast density in young women: A prospective cohort study. Breast Cancer Res. 2019. doi:10.1186/s13058-019-1209-x

69. Ghosh K, Vachon CM, Pankratz VS, et al. Independent association of lobular involution and mammographic breast density with breast cancer risk. J Natl Cancer Inst. 2010;102(22):1716-1723. doi:10.1093/jnci/djq414

70. Rawat RR, Ruderman D, Macklin P, Rimm DL, Agus DB. Correlating nuclear morphometric patterns with estrogen receptor status in breast cancer pathologic specimens. npj Breast Cancer. 2018;4:32. doi:10.1038/s41523-018-0084-4

71. Guo C, Sung H, Zheng S, et al. Age-related terminal duct lobular unit involution in benign tissues from Chinese breast cancer patients with luminal and triple-negative tumors. Breast Cancer Res. 2017;19(1):61. PMCID: PMC5445352. doi:10.1186/s13058-017-0850-5

72. Yang XR, Figueroa JD, Falk RT, et al. Analysis of terminal duct lobular unit involution in luminal A and basal breast cancers. Breast Cancer Res. 2012;14(2):R64. PMCID: PMC3446399. doi:10.1186/bcr3170

73. Sung H, Guo C, Li E, et al. The relationship between terminal duct lobular unit features and mammographic density among Chinese breast cancer patients. Int J Cancer. 
medRxiv preprint doi: https://doi.org/10.1101/2020.09.02.20186643; this version posted September 3, 2020. The copyright holder for this preprint

(which was not certified by peer review) is the author/funder, who has granted medRxiv a license to display the preprint in perpetuity. It is made available under a CC-BY-NC-ND 4.0 International license.

2019;145(1):70-77. doi:10.1002/ijc.32077

74. Gierach GL, Patel DA, Pfeiffer RM, et al. Relationship of terminal duct lobular unit involution of the breast with area and volume mammographic densities. Cancer Prev Res. 2016;9(2):149-158. doi:10.1158/1940-6207.CAPR-15-0282

75. Shekhar MPV, Werdell J, Santner SJ, Pauley RJ, Tait L. Breast stroma plays a dominant regulatory role in breast epithelial growth and differentiation: Implications for tumor development and progression. Cancer Res. 2001;61(4):1320-1326. 
medRxiv preprint doi: https://doi.org/10.1101/2020.09.02.20186643; this version posted September 3, 2020. The copyright holder for this preprint (which was not certified by peer review) is the author/funder, who has granted medRxiv a license to display the preprint in perpetuity. It is made available under a CC-BY-NC-ND 4.0 International license .

Table 1. Benign breast disease (BBD) nested case-control study participants.

\begin{tabular}{|c|c|c|}
\hline & Cases, $n(\%)$ & Controls, $n(\%)$ \\
\hline$n$ & 293 & 1132 \\
\hline \multicolumn{3}{|l|}{ Age at BBD biopsy } \\
\hline$<40$ years & $75(25.6)$ & $251(22.2)$ \\
\hline $40-49$ years & $131(44.7)$ & $438(38.7)$ \\
\hline $50-59$ years & $61(20.8)$ & $293(25.9)$ \\
\hline$\geq 60$ years & $26(8.9)$ & $150(13.3)$ \\
\hline \multicolumn{3}{|l|}{ Year of BBD biopsy } \\
\hline Before 1970 & $30(10.2)$ & 55 (4.9) \\
\hline 1970 to 1979 & $77(26.3)$ & $226(20.0)$ \\
\hline 1980 to 1989 & $129(44.0)$ & $501(44.3)$ \\
\hline After 1989 & $57(19.4)$ & 350 (30.9) \\
\hline \multicolumn{3}{|l|}{ Age at breast cancer diagnosis/index date } \\
\hline$<45$ years & $41(14.0)$ & $206(18.2)$ \\
\hline $45-54$ years & $110(37.5)$ & $371(32.8)$ \\
\hline$\geq 55$ years & $142(48.5)$ & $555(49.0)$ \\
\hline \multicolumn{3}{|l|}{ Years between BBD biopsy and breast cancer diagnosis/index date } \\
\hline 0.5 to 4.9 years & $90(30.7)$ & $532(47.0)$ \\
\hline 5.0 to 9.9 years & $103(35.2)$ & $286(25.3)$ \\
\hline 10.0 to 14.9 years & $54(18.4)$ & $173(15.3)$ \\
\hline$\geq 15.0$ years & $46(15.7)$ & $141(12.4)$ \\
\hline \multicolumn{3}{|l|}{ BBD histological subtype } \\
\hline Non-proliferative & $63(21.50)$ & $331(29.2)$ \\
\hline Proliferative without atypia & $148(50.5)$ & $645(57.0)$ \\
\hline Atypical hyperplasia & $82(28.0)$ & $156(13.8)$ \\
\hline
\end{tabular}


Table 2. Tissue composition (\%) and breast cancer risk factors among 1132 controls. Data presented for age are means (95\% confidence interval). Data for other variables are presented as age-adjusted means ( $95 \%$ confidence interval); age was adjusted as a continuous variable.

\begin{tabular}{|c|c|c|c|c|}
\hline & $n$ & Epithelium (\%) & Fibrous stroma (\%) & Fat (\%) \\
\hline \multicolumn{5}{|l|}{ Age at BBD biopsy } \\
\hline$<40$ years & 251 & $9.2(8.5,10.0)$ & $76.0(74.3,77.7)$ & $7.8(6.9,8.8)$ \\
\hline $40-49$ years & 438 & $7.8(7.3,8.3)$ & $72.0(70.8,73.2)$ & $13.2(12.0,14.4)$ \\
\hline $50-59$ years & 293 & $6.1(5.7,6.6)$ & $69.1(67.7,70.5)$ & $17.5(15.6,19.6)$ \\
\hline$\geq 60$ years & 150 & $5.0(4.5,5.6)$ & $63.5(61.7,65.4)$ & $23.9(20.4,28.0)$ \\
\hline$p$-value & & $<0.001$ & $<0.001$ & $<0.001$ \\
\hline \multicolumn{5}{|l|}{ BBD histological subtype } \\
\hline Non-proliferative & 331 & $5.7(5.3,6.1)$ & $68.3(67.0,69.7)$ & $16.2(14.6,18.0)$ \\
\hline Proliferative without atypia & 645 & $7.8(7.5,8.2)$ & $71.8(70.9,72.8)$ & $12.5(11.6,13.5)$ \\
\hline Atypical hyperplasia & 156 & $8.0(7.2,8.8)$ & $72.8(70.8,74.8)$ & $13.3(11.4,15.6)$ \\
\hline$p$-value & & $<0.001$ & $<0.001$ & $<0.001$ \\
\hline \multicolumn{5}{|l|}{ Body size at ages $5-10$ years } \\
\hline Level 1 & 322 & $7.5(7.0,8.0)$ & $72.0(70.6,73.4)$ & $12.5(11.2,14.0)$ \\
\hline Level 1.5 to 2 & 290 & $7.1(6.6,7.7)$ & $71.8(70.4,73.3)$ & $12.8(11.4,14.3)$ \\
\hline Level $\geq 2.5$ & 367 & $7.0(6.5,7.5)$ & $69.9(68.6,71.2)$ & $14.6(13.2,16.2)$ \\
\hline$p$-value & & 0.42 & 0.048 & 0.09 \\
\hline \multicolumn{5}{|l|}{ Body mass index $\left(\mathrm{kg} / \mathrm{m}^{2}\right)$} \\
\hline$<25$ & 641 & $7.1(6.8,7.5)$ & $72.6(71.6,73.6)$ & $12.3(11.4,13.3)$ \\
\hline 25 to $<30$ & 303 & $7.3(6.7,7.8)$ & $70.7(69.3,72.1)$ & $13.5(12.1,15.0)$ \\
\hline$\geq 30$ & 173 & $7.2(6.5,7.9)$ & $65.5(63.8,67.2)$ & $19.8(17.1,22.8)$ \\
\hline$p$-value & & 0.91 & $<0.001$ & $<0.001$ \\
\hline \multicolumn{5}{|l|}{ Age of menarche } \\
\hline$\leq 12$ years & 532 & $7.0(6.6,7.4)$ & $70.0(68.9,71.0)$ & $14.6(13.5,15.9)$ \\
\hline 13 years & 335 & $7.2(6.7,7.7)$ & $71.1(69.7,72.5)$ & $12.5(11.3,13.9)$ \\
\hline$\geq 14$ years & 260 & $7.4(6.9,8.1)$ & $72.8(71.2,74.4)$ & $13.0(11.6,14.7)$ \\
\hline$p$-value & & 0.50 & 0.01 & 0.05 \\
\hline \multicolumn{5}{|l|}{ Parity } \\
\hline Nulliparous & 107 & $5.2(4.6,5.9)$ & $73.8(71.3,76.4)$ & $9.7(8.1,11.7)$ \\
\hline Parous & 1020 & $7.4(7.1,7.7)$ & $70.6(69.8,71.4)$ & $14.2(13.3,15.0)$ \\
\hline$p$-value & & $<0.001$ & 0.02 & $<0.001$ \\
\hline \multicolumn{5}{|l|}{ Number of births } \\
\hline Nulliparous & 107 & $5.8(5.1,6.7)$ & $75.8(73.2,78.5)$ & $8.1(6.7,9.9)$ \\
\hline Primiparous (1 birth) & 97 & $7.0(6.1,8.1)$ & $73.4(70.8,76.2)$ & $12.6(10.3,15.5)$ \\
\hline Multiparous ( $\geq 2$ births) & 923 & $7.3(7.0,7.7)$ & $70.1(69.3,71.0)$ & $14.6(13.7,15.6)$ \\
\hline$p$-value & & 0.005 & $<0.001$ & $<0.001$ \\
\hline \multicolumn{5}{|l|}{ Time between last birth and BBD biopsy } \\
\hline 0 years (i.e., nulliparous) & 107 & $5.2(4.6,5.9)$ & $73.7(71.2,76.3)$ & $9.8(8.1,11.8)$ \\
\hline$<20$ years (among parous women) & 578 & $7.6(7.1,8.0)$ & $70.4(69.2,71.5)$ & $15.1(13.8,16.5)$ \\
\hline$\geq 20$ years (among parous women) & 409 & $7.0(6.5,7.5)$ & $70.3(68.8,71.8)$ & $14.3(12.8,16.1)$ \\
\hline$p$-value & & $<0.001$ & 0.04 & $<0.001$ \\
\hline \multicolumn{5}{|l|}{ Age at first birth among parous women } \\
\hline$<25$ years & 563 & $7.1(6.8,7.5)$ & $70.5(69.4,71.5)$ & $14.8(13.8,15.8)$ \\
\hline 25 to 29 years & 359 & $7.7(7.2,8.2)$ & $69.6(68.3,70.9)$ & $14.4(13.2,15.7)$ \\
\hline$\geq 30$ years & 101 & $7.1(6.3,8.1)$ & $72.8(70.3,75.4)$ & $12.8(10.9,15.0)$ \\
\hline$p$-value & & 0.19 & 0.08 & 0.27 \\
\hline
\end{tabular}

Birth index among parous women 
medRxiv preprint doi: https://doi.org/10.1101/2020.09.02.20186643; this version posted September 3, 2020. The copyright holder for this preprint (which was not certified by peer review) is the author/funder, who has granted medRxiv a license to display the preprint in perpetuity. It is made available under a CC-BY-NC-ND 4.0 International license.

\begin{tabular}{rcccc}
$\leq 30$ & 229 & $7.4(6.8,8.1)$ & $72.0(70.3,73.8)$ & $13.3(11.9,14.9)$ \\
31 to 59 & 281 & $7.7(7.2,8.3)$ & $71.5(70.0,72.9)$ & $13.8(12.6,15.2)$ \\
$\geq 60$ & 231 & $7.8(7.2,8.6)$ & $70.9(69.2,72.6)$ & $13.5(12.1,15.0)$ \\
$p$-value & & 0.65 & 0.67 & 0.85 \\
Breastfeeding among parous women & & & & \\
Never & 409 & $7.1(6.7,7.5)$ & $70.2(69.0,71.4)$ & $15.3(14.1,16.5)$ \\
$<6$ months & 209 & $7.3(6.7,8.0)$ & $70.9(69.2,72.6)$ & $15.4(13.8,17.2)$ \\
$\geq 6$ months & 305 & $7.5(7.0,8.1)$ & $70.4(69.0,71.8)$ & $13.5(12.3,14.8)$ \\
$p$-value & & 0.47 & 0.79 & 0.09 \\
Menopausal Status & & & & \\
& & & & \\
Pre & 679 & $7.8(7.3,8.2)$ & $72.4(71.2,73.5)$ & $13.2(12.0,14.4)$ \\
Post & 365 & $6.3(5.7,6.9)$ & $68.8(67.2,70.6)$ & $13.7(11.9,15.7)$ \\
$p$-value & & $\mathbf{0 . 0 0 1}$ & $\mathbf{0 . 0 0 4}$ & 0.71 \\
\hline
\end{tabular}


medRxiv preprint doi: https://doi.org/10.1101/2020.09.02.20186643; this version posted September 3, 2020. The copyright holder for this preprint (which was not certified by peer review) is the author/funder, who has granted medRxiv a license to display the preprint in perpetuity.

It is made available under a CC-BY-NC-ND 4.0 International license .

Table 3. The association between tissue composition and breast cancer risk was evaluated using unconditional logistic regression models to estimate odd ratios (ORs) and $95 \%$ confidence intervals $(\mathrm{Cl})$.

\begin{tabular}{|c|c|c|c|c|c|}
\hline & Quartile 1 & Quartile 2 & Quartile 3 & Quartile 4 & p-trend \\
\hline \multicolumn{6}{|l|}{ Epithelium } \\
\hline Cases/Controls, $n$ & $56 / 283$ & $65 / 283$ & $68 / 283$ & $104 / 283$ & \\
\hline Quartile cutoff, \% & $<4.8$ & $\geq 4.8$ to $<7.5$ & $\geq 7.5$ to $<11.2$ & $\geq 11.2$ & \\
\hline Model 1 & Ref & $1.12(0.76,1.67)$ & $1.12(0.75,1.67)$ & $1.53(1.04,2.27)$ & 0.02 \\
\hline Model 2 & Ref & $0.95(0.63,1.43)$ & $0.92(0.61,1.39)$ & $1.36(0.91,2.03)$ & 0.06 \\
\hline Model 3 & Ref & $0.95(0.61,1.49)$ & $0.95(0.61,1.49)$ & $1.39(0.91,2.14)$ & 0.047 \\
\hline \multicolumn{6}{|l|}{ Fibrous stroma } \\
\hline Cases/Controls, $n$ & $62 / 283$ & $67 / 283$ & $78 / 283$ & $86 / 283$ & \\
\hline Quartile cutoff, \% & $<64.5$ & $\geq 64.5$ to $<73.5$ & $\geq 73.5$ to $<81.3$ & $\geq 81.3$ & \\
\hline Model 1 & Ref & $0.98(0.66,1.45)$ & $1.07(0.73,1.57)$ & $1.20(0.81,1.76)$ & 0.33 \\
\hline Model 2 & Ref & $0.87(0.58,1.30)$ & $0.96(0.65,1.42)$ & $1.07(0.72,1.59)$ & 0.65 \\
\hline Model 3 & Ref & $0.78(0.51,1.20)$ & $0.86(0.56,1.31)$ & $0.93(0.61,1.41)$ & 0.85 \\
\hline \multicolumn{6}{|l|}{ Fat } \\
\hline Cases/Controls, $n$ & $102 / 283$ & $80 / 283$ & $49 / 283$ & $62 / 283$ & \\
\hline Quartile cutoff, \% & $<8.7$ & $\geq 8.7$ to $<16.7$ & $\geq 16.7$ to $<27.0$ & $\geq 27.0$ & \\
\hline Model 1 & Ref & $0.81(0.57,1.15)$ & $0.55(0.36,0.81)$ & $0.75(0.50,1.12)$ & 0.11 \\
\hline Model 2 & Ref & $0.81(0.56,1.15)$ & $0.55(0.36,0.82)$ & $0.83(0.55,1.25)$ & 0.27 \\
\hline Model 3 & Ref & $0.83(0.58,1.21)$ & $0.56(0.36,0.85)$ & $0.93(0.59,1.45)$ & 0.52 \\
\hline
\end{tabular}

Each tissue region was categorized into quartiles as defined by the distribution among the controls. Model 1 adjusted for matching factors. Model 2 adjusted for matching factors and BBD histological subtypes. Model 3 adjusted for matching factors, BBD histological subtypes, parity, menopausal status, and BMI. The median value for each quartile was included as a continuous variable in the unconditional logistic regression for Model 1, 2, and 3, to obtain the $p$-trend value (Wald test). 


\section{Figure Legends}

Figure 1. Overview of our BBD image analysis pipeline. (A) A whole slide image (WSI). (B) Image processing to extract tissue-containing areas of the WSI. (C) Applying our tissue segmentation and nuclei detection networks created in the development phase to a WSI to obtain a segmentation map. (D) From the segmentation map, computer-derived morphometric features were extracted. Percentages of tissue regions were also computed from the map. Morphometric data were summarized from all WSIs belonging to the same woman. (E) Identifying if morphometric features are associated with breast cancer.

Figure 2. An example of an original image, ground truth, and automated segmentation or detection for each deep-learning network. (A) For tissue segmentation, white represents background, green represents fibrous stroma, red is epithelium, and purple is fat. (B) For cell nuclei detection, white represents background, red is nucleus, and cyan is nuclei membrane border. The final output produces a binary mask which considers nucleus membrane pixels to be part of the background.

Figure 3. Boxplots display the amount of each tissue region (\%) among cases and controls (A), and when stratified by benign breast disease (BBD) histological subtypes (B, C, D). * denotes $p<0.05$ and ** denotes $p<0.001$.

Supplementary Figure 1. A schematic diagram showing how four patches were selected on a whole slide image for manual annotation. The dark areas of the whole slide image correspond to areas of the slide that do not contain tissue and were thus not scanned by the scanner.

Supplementary Figure 2. Example patches used to train the tissue segmentation and nuclei detection networks. The top row in each section display the original patches, the bottom row contains the corresponding manually annotated ground truths. Images display a broad spectrum of staining colors and intensities. (A) Examples of tissue segmentation training patches from the Nurses' Health Studies benign breast disease incidence study. In our ground truth annotations, white represents background, green represents fibrous stroma, red is epithelium, and purple is fat. (B) Examples of nuclei detection training patches from invasive breast cancer cases from The Cancer Genome Atlas. In the ground truth annotations, white represents background, red is nucleus, and cyan is nuclei membrane border. 

A. Tissue segmentation network performance

Original Image

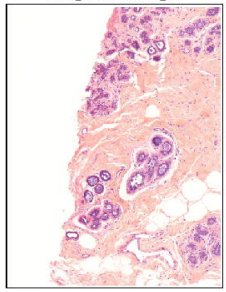

B. Cell nuclei detection network performance
Original Image

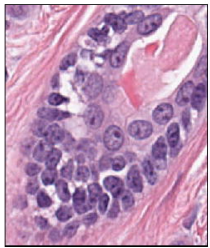

Ground truth

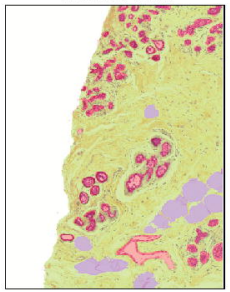

Automated Segmentation

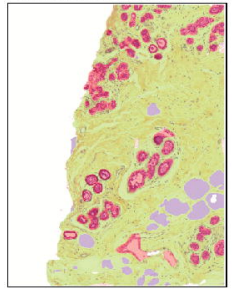

Automated Detection

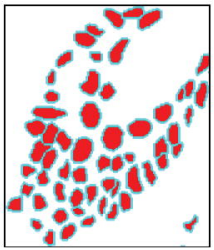


Example of an whole slide image containing a radial scar (i.e., proliferative without atypia subtype)
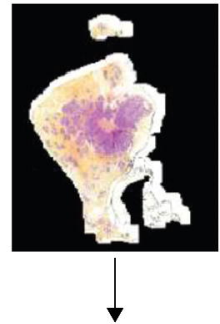

Example of a patch

Example of a patch with manual annotation
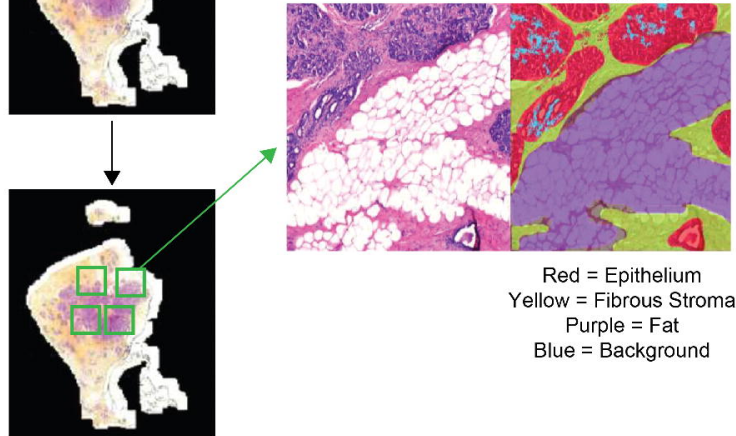

Red = Epithelium

Yellow = Fibrous Stroma

Purple = Fat Blue $=$ Background

Example of 4 selected patches (green squares). Each patch of $2048 \times 2048$ pixel encompasses epithelium, fibrous stroma, fat, and background. 

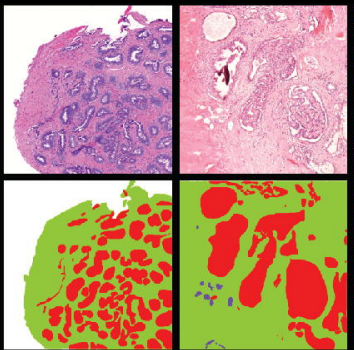
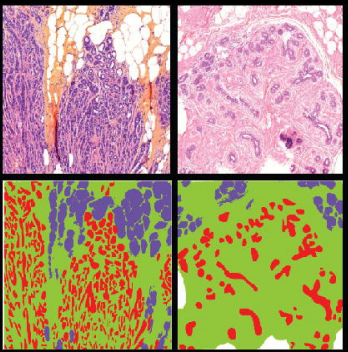
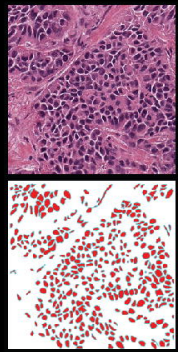
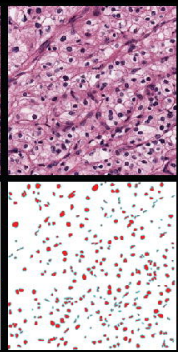
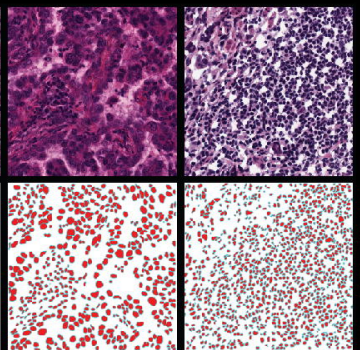

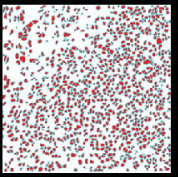

